\title{
MOLECULAR GENETIC ANALYSIS OF VARIABILITY \\ OF HA, NA AND NPGENES OF INFLUENZA VIRUS \\ (COMPARED TO H1N1 AND H7N9 STRAINS)
}

\section{Buriachenko Semen ${ }^{1}$}

DOI: https://doi.org/10.30525/978-9934-571-89-3_78

Virus Influenza virus (the genus Orthomyxoviridae) is an avian influenza agent an acute highly contagious disease of the respiratory and gastrointestinal tract [1, p. 12]. According to polymorphism, the nucleotide and amino acid sequences of Influenza virus are divided into three types: A, B and C. The most common zoonotic agent is a type $\mathrm{A}$ virus that is able to overcome the interspecific barrier and affect birds and mammals, including humans [2, p. 24; 3, p. 41]. Type A virus has the largest number of HBs among the Influenza virus. Such properties of this virus are due to the higher speed of its evolution than those of type B and C [1, p. 5; 3, p. 78]. Periodically, Influenza A causes epizootics, epidemics and pandemics [3, p. 66]. In recent years, due to mass epizootics in more than 50 countries, the forced slaughter of millions of heads of birds was carried out $[4$, p. 12; 5, p. 4]. The most important factors in the virulence of the avian influenza virus are surface proteins hemagglutinin $(H A$, or $H)$ and neurominidase $(N A$ or $N)$, and a replication factor of a nucleoprotein $(N P)$. Polymorphism of these genetic data remains insufficiently investigated $[6$, p. $58 ; 7$, p. $98 ; 8$, p. 43]. Influenza virusA is divided into subtypes according to the polymorphism of $N A$ and $N A$. There are $18 N A(H)$ and $11 N A(N)$ subtypes. Epizootics are largely due to the highly virulent strains of H1N1 and H7N9 [2, p. 52]. The aim of the study was to investigate the variability of $H A, N A$ and $N P$ genuses of avian influenza viruses encoding the virulence factors of $H A, N A$ and $N P$, respectively, for the $\mathrm{H} 1 \mathrm{~N} 1$ and $\mathrm{H} 7 \mathrm{~N} 9$ strains. The research material used was nucleotide sequences of the $H A, N A$ and $N P$ gene of the avian influenza virus strains H1N1 and H7N9, obtained from the National Center for Biotechnology Information (National Center for Biotechnology Information). Cluster analysis and genetic sequencing of $H A, N A$ and $N P$ gene sequences were performed using the MEGA 6 program using the ClustalW algorithm. The dendrograms were constructed using a pairwise clustering method with arithmetic averaging (UnweightedPairGroupMethod), the reliability was counted using bootstrap analysis with the number of replicates equal to 500. The result was found to be greater than 70 . Validity of the $H A, N A$ and $N P$ genes was investigated by local alignment of the selected sequences using the Smith-Waterman algorithm using the Vector NTI-11 program. Polymorphic locus was determined on the longest nucleotide sequences of the corresponding genes. Numbers on nodes is an indicator of bootstrap analysis. On the X-axis - the length of the branches (replacing the position). As a result of the cluster analysis of the sequence of the HA gene for H1N1 and H7N9 strains, separate clusters form. The

\footnotetext{
${ }^{1}$ NSC Institute of Experimental and Clinical Veterinary Medicine, Ukraine 
nucleotide sequences of the H1N1 and H7N9 strains of the gene form separate clusters, indicating a high level of polymorphism of this gene. The NA sequence of the H7N9 strain forms a separate cluster, as well as a common cluster with sequences of the H1N1 strain. The nucleotide sequences of the H1N1 strain gene are genetically identical. A portion of the NA nucleotide sequence of the H7N9 strain forms a separate cluster, the other part forming a joint cluster with nucleotide sequences of the NA gene H1N1 strain. The sequence of the NP gene of the H1N1 strain forms a separate cluster, as well as a common cluster with sequences of the H7N9 strain. The nucleotide sequences of the $N P$ genype H7N9 strain and part of the nucleotide sequences of the H1N1 strain are genetically identical. A part of the nucleotide sequence of the $N P$ gene of the H1N1 strain forms a separate cluster, the other part forming a joint cluster with the nucleotide sequences of the $N A$ gene H7N9. Thus, it has been shown that the $H A$ gene of the avian influenza virus has a greater cross-site polymorphism than the $N A$ and $N P$ genes. The polymorphism of the $H A$ gene is higher in the strain of the $\mathrm{H} 1 \mathrm{~N} 1$ avian influenza virus, the $N A$ gene in $\mathrm{H} 7 \mathrm{~N} 9$, the $N P$ gene in H1N1. The bootstrap analysis in all cases is greater than 70 , indicating the reliability of the results. According to the results of the alignment, the most variable genome is $N A$, the least variable is $N P$, which coincides with the result of the cluster analysis. In all cases, the most common polymorphism is single-nucleotide substitution, while the most polymorphic regions are located at the 3 'and 5' ends of the sequences. It is likely that the high variability of the $H A$ gene, and somewhat lower, of $N A$, causes the ability of the avian influenza virus, in particular its highstrain $\mathrm{H} 1 \mathrm{~N} 1$ and H7N9 strains, to overcome the interspecific barrier, while the replication factor encoded by the $N P$ genome is less important for overcoming the interspecific bar which determines its lower, versus $N A$ and $N A$ variation.

1. Alignment and cluster analysis of $H A, N A$ and $N P$ genes of avian influenza virus type A.

2. Showing the variability of these genes compared to H1N1 and H7N9 strains.

3 . The constructed dendrograms show the degree of variability of the studied genes inside and between strains.

4. The alignment results coincide with the result of a cluster analysis of the nucleotide sequences of the $H A, N A$ and $N P$ genes.

5. The polymorphic sites and the type of polymorphism of the studied genes were determined.

6 . The results of the study can be used in the study of phylogeny and molecular evolution

\section{References:}

1. Taubenberger J. K. Influenza Virus Evolution, Host Adaptation and Pandemic Formation / J. K. Taubenberger, J. C. Kash // Cell Host Microbe. - 2010. - № 7 (6). - P. 440-451.

2. Ozawa M. Crosstalk between animal and human influenza viruses / M. Ozawa, Y. Kawaoka // Annu Rev AnimBiosci. - 2013. - № 1. - P. 21-42.

3. Webster R. G. Evolution and ecology of influenza A viruses. / R. G. Webster, W. J. Bean, O. T. Gorman, T. M. Chambers, Y. Kawaoka // Microbiol Rev. - 1992. - № 56 (1). - P. 152-179. 
4. Staats C. B. Diversity of influenza viruses in swine and the emergence of a novel human pandemic influenza A (H1N1). / C. B. Staats, R. G. Webster, R. J. Webby // Influenza Other Respir Viruses. - 2009. - № 3(5). - P. 207-213.

5. Allison A. B. Cyclic Avian Mass Mortality in the Northeastern United States Is Associated with a Novel Orthomyxovirus // A. B. Allison, J. R. Ballard, R. B. Tesh, J. D. Brown, M. G. Ruder, M. K. Keel, B. A. Munk,R.1 M. Mickley, S. E. J. Gibbs, A. P. A. Travassos da Rosa, J. C. Ellis, H. S. Ip, V. I. Shearn-Bochsler, M. B. Rogers, E. Ghedin, E. C. Holmes, C. R. Parrish, C. Dwyerj // J Virol. - 2015. - № 89(2). - P. 1389-1403.

6. Dukhovlinov I. Study of immunogenicity of recombinant proteins based on hemagglutinin and neuraminidase conservative epitopes of Influenza A virus / I. Dukhovlinov, R. Al-Shekhadat, E. Fedorova, L. Stepanova, M. Potapchuk, I. Repko, O. Rusova, A. Orlov, L. Tsybalova, O. Kiselev // Med SciMonit Basic Res. - 2013. - № 19. - P. 221-227.

7. Wu C. Influenza A surface glycosylation and vaccine design. / C.Wu, C. Lin,T. Tsai, C. Lee, H. Chuang, J. Chen, M. Tsai, B. Chen, P. Lo, C. Liu, a V. Shivatare, C. Wong // Proc Natl AcadSci U S A. - 2017. - № 114 (2). - P. 280-285.

8. Tada T. Emergence of Avian Influenza Viruses with Enhanced Transcription Activity by a Single Amino Acid Substitution in the Nucleoprotein during Replication in Chicken Brains // T. Tada, K. Suzuki, Y. Sakurai, M. Kubo, H. Okada, T. Itoh, K. Tsukamoto // J Virol. - 2011. № 85 (19). - P. 10354-10363. 